\title{
Positive QRS Complex in Lead I as a Malignant Sign in Right Ventricular Outflow Tract Tachycardia
}

\author{
- Comparison Between Polymorphic and \\ Monomorphic Ventricular Tachycardia -
}

Kenji Kurosaki, MD; Akihiko Nogami, MD; Yasuhiro Shirai, MD; Shinya Kowase, MD

\begin{abstract}
Background: Idiopathic ventricular fibrillation (VF) or polymorphic ventricular tachycardia (PVT) arising from the right ventricular outflow tract (RVOT) is occasionally observed. The difference in the initial ventricular premature contraction (VPC) between VF/PVT and monomorphic VT (MVT) from the RVOT, however, has not yet been fully investigated.
\end{abstract}

\begin{abstract}
Methods and Results: The electrocardiogram findings and the clinical characteristics were compared between 14 patients with PVT and 77 with MVT. The episodes of syncope were more frequent in the VF and/or PVT group (57\%) than in the MVT group (10\%). An initial VPC with a positive QRS complex in lead I was observed in 10 (71\%) of 14 patients with VF/PVT, and in $27(35 \%)$ of 77 patients with MVT $(\mathrm{P}<0.05)$. Although radiofrequency $(\mathrm{RF})$ catheter ablation targeting the trigger VPC often produced a morphological change, VF/PVT was eliminated in 13 (93\%) of 14 patients after additional RF applications.
\end{abstract}

Conclusions: Malignant arrhythmias from the RVOT, although rare, should be considered when the patient has a syncopal episode and VPC with a positive QRS complex in lead I. (Circ J 2013; 77: 968-974)

Key Words: Outflow tract; Polymorphic ventricular tachycardia; QRS morphology; Radiofrequency catheter ablation; Ventricular fibrillation

$\mathbf{V}$ entricular premature contraction (VPC) and monomorphic ventricular tachycardia (MVT) arising from the right ventricular outflow tract (RVOT) without any structural heart disease are considered to be benign. In contrast, malignant arrhythmias such as ventricular fibrillation (VF) and polymorphic ventricular tachycardia (PVT) from the RVOT are commonly observed in patients with arrhythmogenic right ventricular cardiomyopathy (ARVC) or Brugada syndrome. But even in the normal heart, VF and PVT initiated by RVOT-VPC are occasionally observed. ${ }^{1-9}$ Previous reports of idiopathic RVOT-VF and/or PVT indicated that the QRS waveform of the VPC initiating the VF/PVT was identical to that of the spontaneous VPCs, and radiofrequency catheter ablation (RFCA) targeting the trigger VPC could successfully eliminate the VF/PVT. ${ }^{3-9}$ Although several cases have been reported, no study has referred to the VPC morphology initiating the VF/PVT, and the clinical features of the RVOT-VF/PVT have not been fully investigated.

The aim of this study was to determine whether the positive QRS complex in lead I was associated with RVOT-VF/PVT in comparison with MVT.

\section{Methods}

\section{Subjects}

Between January 2000 and February 2011, 91 patients (29 men, 62 women; mean age, $47 \pm 14$ years; range 16-74 years) who underwent RFCA with symptomatic VF/PVT or MVT arising from the RVOT were included in this study. Patients suspected of having Brugada syndrome $(n=1)$, long QT syndrome $(n=2)$ or ARVC $(n=2)$ were excluded. Patients who had right and left ventricular enlargement $(n=6)$ on computed tomography (CT) or magnetic resonance imaging (MRI) were also excluded. All 91 patients had a normal electrocardiogram (ECG) during sinus rhythm and spontaneous VPCs with a left bundle branch morphology and inferior axis. No patient had any negative $\mathrm{T}$ waves in the $\mathrm{V}_{2}$ or $\mathrm{V}_{3}$ leads of the 12-lead ECG or late potentials in the signal-averaged ECG (SAECG).

PVT was defined as a VT with $>5$ consecutive different $Q R S$ complexes in the surface ECG. VF was defined as ventricular arrhythmia (VA) consisting of disorganized waveforms with hemodynamic decompensation requiring direct cardioversion for termination. Among the 91 patients, VF/PVTs were ob-

Received July 4, 2012; revised manuscript received October 14, 2012; accepted November 20, 2012; released online December 14,

2012 Time for primary review: 16 days

Department of Heart Rhythm Management, Yokohama Rosai Hospital, Yokohama, Japan

Mailing address: Akihiko Nogami, MD, Department of Heart Rhythm Management, Yokohama Rosai Hospital, 3211 Kozukue, Kohoku-

ku, Yokohama 222-0036, Japan. E-mail: akihiko-ind@umin.ac.jp

ISSN-1346-9843 doi:10.1253/circj.CJ-12-0864

All rights are reserved to the Japanese Circulation Society. For permissions, please e-mail: cj@j-circ.or.jp 
Patient 1

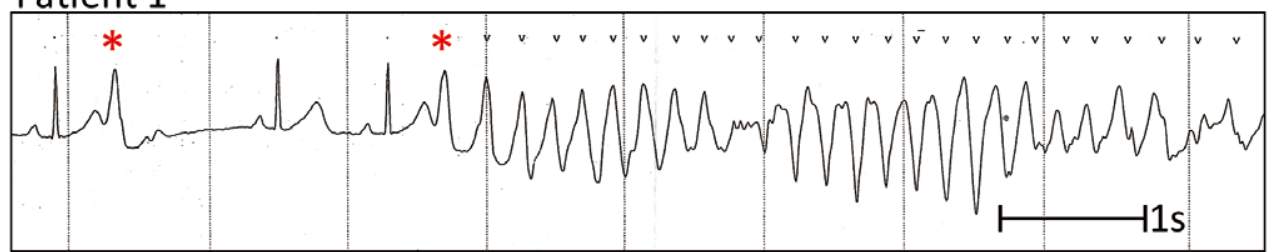

Patient 2

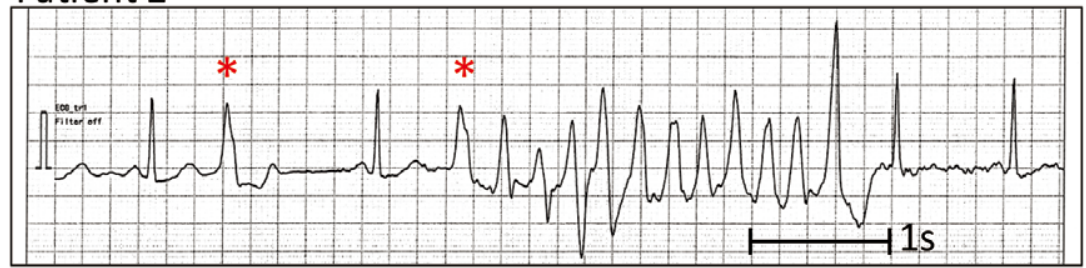

Patient 12

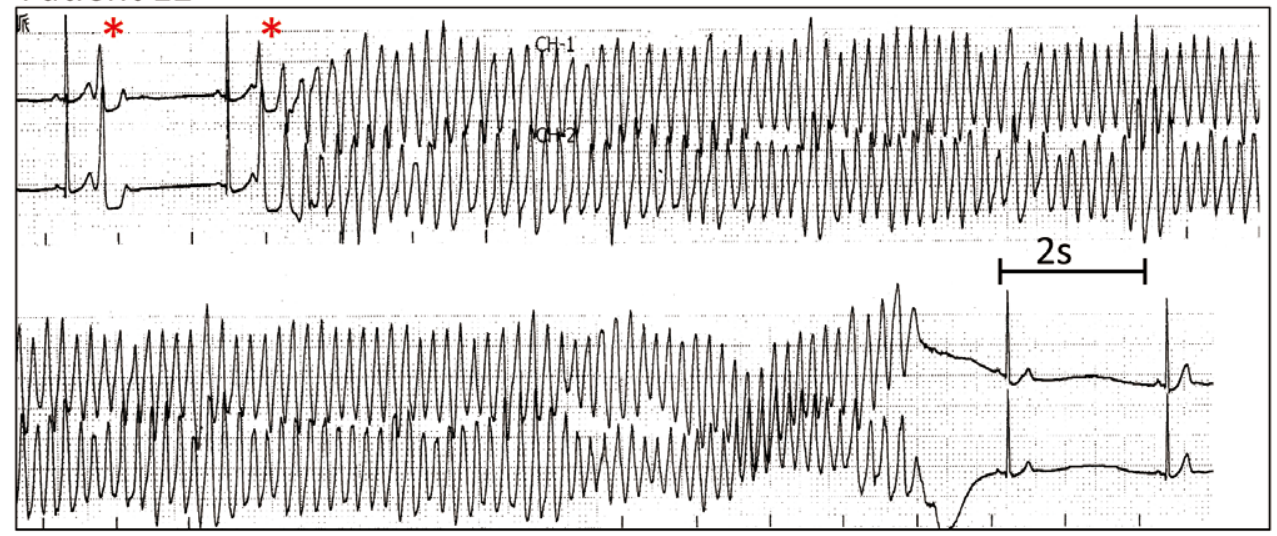

Figure 1. Representative electrocardiogram of polymorphic ventricular tachycardia (PVT) recorded by ambulatory monitoring in 3 patients. The QRS morphology of the first ventricular premature contraction (VPC) initiating PVT was identical to that of the preceding isolated VPC. The QRS morphology of the first VPC initiating PVT was identical to that of the preceding isolated VPC (asterisks).

served in 14 patients and MVT in 77 patients. This study was approved by the institutional ethics committee, and all patients provided written informed consent to participate.

\section{Analysis of 12-Lead ECG}

We confirmed that the first VPC initiating the VF/PVT was identical to the frequent isolated VPCs (Figure 1) and analyzed the VPC morphology in each of the 12 leads. In addition, we measured the essential ECG parameters, such as the coupling interval of the first VPC initiating VA and the shortest R-R interval during the VA. A positive QRS complex was defined as a positive deflection exceeding the negative component by $>0.1 \mathrm{mV}$ in amplitude.

\section{Mapping and RFCA}

After anti-arrhythmic agents had been withdrawn for $>5$ halflives, electrophysiological study (EPS) and RFCA were carried out. ${ }^{10}$ The electroanatomical voltage mapping was performed in 29 (32\%) of 91 patients. To induce VA, programmed ventricular stimulation using up to 3 extrastimuli and incremental burst pacing at a cycle length of up to 2:1 ventricular or atrial capture were repeatedly performed from the right atrium and right ventricular apex. In patients without VPCs despite the programmed electrical stimulation in the EP laboratory, i.v. infusion of isoproterenol $(0.5-2.0 \mu \mathrm{g} / \mathrm{min})$ and/or a single injection of phenylephrine $(250 \mu \mathrm{g})$ were given to evoke the VAs. RF energy was delivered under the guidance of activation mapping and pace mapping. If the VPC morphology changed during the procedure, additional RF applications were given to eliminate the VPCs completely.

In RFCA, we used a 7-Fr quadripolar catheter with a nonirrigated 4-mm or 8-mm tip distal electrode, embedded thermistor, interspacing of 2-5-2 mm, and deflectable tip (EP Technologies, San Jose, CA, USA; Biosense-Webster, Diamond Bar, CA, USA; or Japan Lifeline, Tokyo, Japan).

Successful ablation was defined as the elimination of all VT and VPCs without any anti-arrhythmic drugs. Partial success was defined as the elimination of the VT with residual isolated VPCs from the RVOT. A failed ablation was defined as the inability to suppress the VAs.

\section{Statistical Analysis}

Continuous variables are expressed as mean $\pm \mathrm{SD}$, and were compared using Student's t-test. The categorical variables were compared using chi-square analysis. $\mathrm{P}<0.05$ was considered statistically significant. 


\begin{tabular}{|c|c|c|c|c|c|c|c|c|}
\hline & $\begin{array}{c}\text { Age } \\
\text { (years) }\end{array}$ & Gender & $\begin{array}{l}\text { Prior } \\
\text { symptoms }\end{array}$ & $\begin{array}{c}\text { VF } \\
\text { episode }\end{array}$ & $\begin{array}{l}\text { Intrinsic QRS } \\
\text { duration (ms) }\end{array}$ & $\begin{array}{l}\text { Cl of the initial } \\
\text { VPC (ms) }\end{array}$ & $\begin{array}{l}\text { Shortest R-R } \\
\text { during VT (ms) }\end{array}$ & $\begin{array}{l}\text { Total VPCs in the } \\
\text { Holter (/day) }\end{array}$ \\
\hline \multicolumn{9}{|l|}{ Patient } \\
\hline 1 & 54 & $\mathrm{~F}$ & Syncope & Yes & 62 & 420 & 180 & 3,766 \\
\hline 2 & 50 & $\mathrm{~F}$ & Syncope & Yes & 92 & 540 & 200 & 7,246 \\
\hline 3 & 34 & $\mathrm{~F}$ & Syncope & Yes & 95 & 350 & 180 & 10,815 \\
\hline 4 & 56 & $\mathrm{~F}$ & Syncope & Yes & 84 & 400 & 190 & 20,183 \\
\hline 5 & 46 & $\mathrm{~F}$ & Syncope & No & 85 & 440 & 180 & 6,486 \\
\hline 6 & 41 & $\mathrm{~F}$ & Syncope & No & 80 & 560 & 240 & 24,213 \\
\hline 7 & 51 & $F$ & Syncope & No & 84 & 500 & 240 & 21,025 \\
\hline 8 & 33 & $\mathrm{~F}$ & Syncope & No & 75 & 640 & 240 & 18,319 \\
\hline 9 & 55 & $\mathrm{~F}$ & Presyncope & No & 76 & 420 & 220 & 24,691 \\
\hline 10 & 25 & $\mathrm{~F}$ & Presyncope & No & 96 & 400 & 250 & NA \\
\hline 11 & 67 & $\mathrm{~F}$ & Presyncope & No & 82 & 405 & 240 & 2,221 \\
\hline 12 & 41 & $\mathrm{~F}$ & Palpitations & No & 77 & 460 & 240 & 38,953 \\
\hline 13 & 34 & M & Palpitations & No & 72 & 520 & 240 & 19,282 \\
\hline 14 & 50 & M & Palpitations & No & 75 & 640 & 280 & 32,045 \\
\hline Mean \pm SD & $45 \pm 11$ & & & & $81 \pm 9$ & $440 \pm 73$ & $224 \pm 34$ & $19 \pm 12 \times 10^{3}$ \\
\hline
\end{tabular}

$\mathrm{Cl}$, coupling interval; NA, not available; PVT, polymorphic ventricular tachycardia; VF, ventricular fibrillation; VPC, ventricular premature contraction; VT, ventricular tachycardia.

\begin{tabular}{|c|c|c|c|}
\hline & $\begin{array}{c}\text { VF/PVT } \\
(n=14)\end{array}$ & $\begin{array}{c}\text { MVT } \\
(n=77)\end{array}$ & P-value \\
\hline \multicolumn{4}{|l|}{ Clinical characteristics } \\
\hline Age (years) & $45 \pm 11$ & $47 \pm 14$ & NS \\
\hline Female & $12(86)$ & $50(65)$ & NS \\
\hline Episodes of syncope & $8(57)$ & $8(10)$ & $<0.001$ \\
\hline $\begin{array}{l}\text { Episodes of syncope or } \\
\text { presyncope }\end{array}$ & $11(79)$ & $30(39)$ & $<0.01$ \\
\hline VF episodes & $4(29)$ & $0(0)$ & NS \\
\hline FH of SCD & $0(0)$ & $1(1)$ & NS \\
\hline $\begin{array}{l}\text { VPCs on the Holter } \\
\left(\times 10^{3 / \text { day }}\right)\end{array}$ & $19 \pm 12$ & $22 \pm 10$ & NS \\
\hline $\begin{array}{l}\text { VA onset from VPC } \\
\text { diagnosis (months) }\end{array}$ & $120 \pm 118$ & $81 \pm 105$ & NS \\
\hline \multicolumn{4}{|l|}{$\begin{array}{l}\text { Baseline 12-lead ECG } \\
\text { findings }\end{array}$} \\
\hline Positive QRS waves in lead I & $10(71)$ & 27 (35) & $<0.05$ \\
\hline $\mathrm{Cl}$ of the initial VPC (ms) & $440 \pm 73$ & $444 \pm 63$ & NS \\
\hline $\begin{array}{l}\text { Shortest R-R interval during } \\
\text { VA (ms) }\end{array}$ & $224 \pm 34$ & $330 \pm 69$ & $<0.0001$ \\
\hline
\end{tabular}

Data given as mean $\pm \mathrm{SD}$ or $\mathrm{n}(\%)$.

$\mathrm{FH}$, family history; MVT, monomorphic ventricular tachycardia; NS, not significant; SCD, sudden cardiac death; VA, ventricular arrhythmia. Other abbreviations as in Table 1.

\section{Results}

\section{Patient Clinical Characteristics}

The clinical characteristics of the patients with VF/PVT are listed in Table 1. Twelve (86\%) of 14 patients were female. Eight $(57 \%)$ of the 14 patients had prior repeated episodes of syncope at rest. Including presyncope, 11 (79\%) of the 14 patients experienced some symptoms associated with a cerebral circulatory disturbance. In $4(29 \%)$ of the 14 patients, spontaneous VFs were recorded, and implantable cardioverter-defibrillators were implanted. All patients had a normal intrinsic
QRS duration (range, 62-96 ms) without right bundle branch block during sinus rhythm. The mean coupling interval of the initial VPC was $440 \pm 73 \mathrm{~ms}$ (range, 350-640ms). The mean of the shortest coupling interval during the PVT was $224 \pm 34 \mathrm{~ms}$ (range, $180-280 \mathrm{~ms}$ ). The total VPCs in the $24-\mathrm{h}$ Holter monitoring ranged from 2,221 to 38,953 beats $(18,788 \pm 12,228$ beats/day). Comparisons of the clinical characteristics between the VF/PVT and MVT groups are given in Table 2. There was no difference in the age, gender or total number of VPCs in a 24-h period. Prior syncopal episodes were observed more often in the VF/PVT group than in the MVT group $(\mathrm{P}<0.001)$. A family history of sudden cardiac death was noted in 1 patient in the MVT group. No significant difference was observed in the time from VPC detection by medical checkup to the clinical onset of VA between the 2 groups.

\section{ECG Characteristics of the First VPC Initiating VF/PVT}

The VPC waveforms in leads I and II initiating VF/PVT in all 14 patients are shown in Figure 2. Before RFCA, 10 (71\%) of 14 patients in the VF/PVT group had spontaneous VPCs with a positive deflection of the QRS complex in lead I (patients $1-3,5,8,10-13$, and 14). Comparisons of the ECG characteristics between the VF/PVT and MVT groups are given in Table 2. A positive QRS complex in lead I was more frequent in the VF/PVT group than in the MVT group $(\mathrm{P}<0.05)$. The positive QRS complex in lead I predicted the occurrence of VF/PVT with a sensitivity of $71 \%$, specificity of $65 \%$, positive predictive accuracy of $27 \%$, and negative predictive accuracy of $93 \%$. We also compared the following parameters: (1) amplitude of the R wave in the inferior leads; (2) duration and amplitude of the $\mathrm{R}$ wave in $\mathrm{V}_{1}$ and $\mathrm{V}_{2}$; (3) presence of QRS notching in the inferior leads; (4) precordial transition zone; (5) $\mathrm{R} / \mathrm{S}$ ratio in lead $\mathrm{V}_{2}$; and (6) $\mathrm{Q}$ wave ratio of $\mathrm{a} \mathrm{V}_{\mathrm{R}} / \mathrm{a} \mathrm{V}_{\mathrm{L}}$. No other significant difference, however, was detected in the QRS waveform during VPCs between the 2 groups. The positive QRS complex in lead I with the syncopal episodes predicted the occurrence of VF/PVT with a sensitivity of $43 \%$, specificity of $99 \%$, positive predictive accuracy of $86 \%$, and negative 


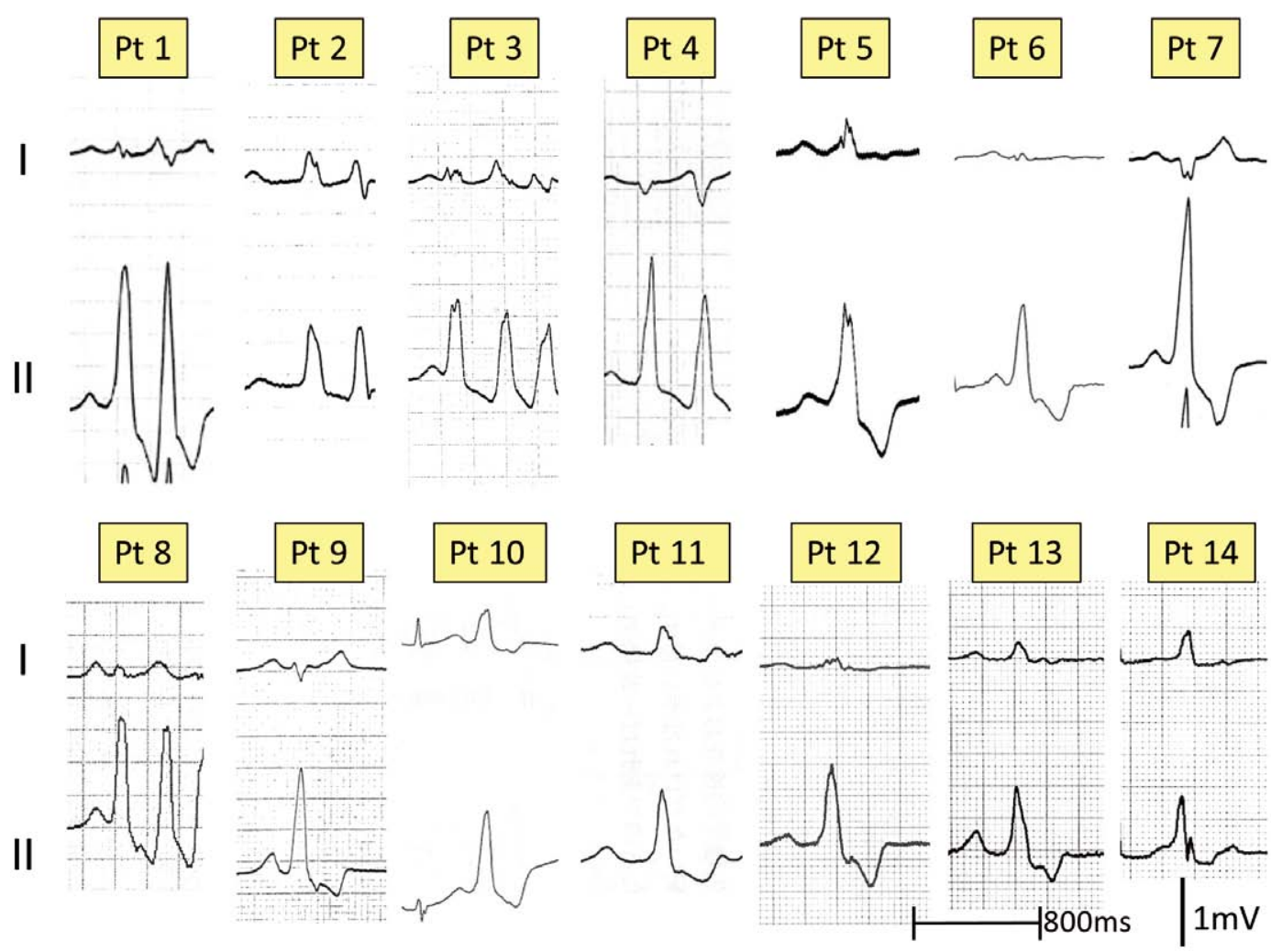

Figure 2. QRS complexes of the initial ventricular premature contraction in leads I and II of all 14 patients with ventricular fibrillation or polymorphic ventricular tachycardia.

predictive accuracy of $90 \%$. The coupling interval of the initial VPC was also unchanged between the 2 groups. The shortest R-R interval during VT was significantly shorter in the VF/ PVT group than in the MVT group $(\mathrm{P}<0.0001)$.

\section{Mapping and RFCA}

The results of EPS and RFCA are given in Table 3. The documented VT was repeatedly inducible in $13(17 \%)$ of 77 patients in the MVT group. In contrast, no VA was inducible during EPS in the VF/PVT groups. In 5 (36\%) of 14 patients with VF/ PVT, however, the documented polymorphic QRS waves were reproduced by burst pacing at the successful ablation sites. A change in the spontaneous VPC morphology after several RF applications was observed in more than half of the patients in both the VF/PVT (71\%) and MVT groups (52\%). At the start of the RFCA, 4 of 14 patients (patients 4, 6, 7, and 9) in the VF/PVT group had spontaneous VPCs with an isoelectric or negative QRS complex in lead I, but 2 of these patients (patients 4 and 6) with non-positive QRS complexes in lead I acquired a positive QRS after several RF energy applications (Figure 3). Such a morphological change was also observed in $7(14 \%)$ of 50 patients in the MVT group with isoelectric or negative QRS complexes in lead I. Including the QRS morphological change of the VPC, a positive QRS complex in lead I was observed in $12(86 \%)$ of 14 patients in the VF/PVT group and in 34 (44\%) of 77 patients in the MVT group. As shown in Figure 4, in the VF/PVT group, the VPCs were finally eliminated at the posterior attachment of the free wall in 7 patients, at the posterior attachment of the septum in 2,

\begin{tabular}{|c|c|c|c|}
\hline & $\begin{array}{l}\text { VF/PVT } \\
(n=14)\end{array}$ & $\begin{array}{c}\text { MVT } \\
(n=77)\end{array}$ & P-value \\
\hline \multicolumn{4}{|l|}{ EPS } \\
\hline ERP of RVA (ms) & $234 \pm 21$ & $248 \pm 22$ & NS \\
\hline Inducibility of documented VT & 0 & $13(17)$ & $<0.05$ \\
\hline \multicolumn{4}{|l|}{ RFCA } \\
\hline $\begin{array}{l}\text { Any morphological change } \\
\text { during RFCA }\end{array}$ & $10(71)$ & $40(52)$ & NS \\
\hline $\begin{array}{l}\text { Morphological change from an } \\
\text { iso/neg to positive QRS in lead I }\end{array}$ & $2(14)$ & $7(9)$ & NS \\
\hline \multicolumn{4}{|l|}{ Short-term results } \\
\hline Success & $13(93)$ & $65(84)$ & NS \\
\hline Partial success & $1(7)$ & $10(13)$ & NS \\
\hline Failure & 0 & $2(3)$ & NS \\
\hline \multicolumn{4}{|l|}{ Long-term results } \\
\hline Follow-up period (months) & $68 \pm 23$ & $71 \pm 36$ & NS \\
\hline VF/VT recurrence & 0 & $2(3)$ & NS \\
\hline Syncope & 0 & 0 & NS \\
\hline Death & 0 & 0 & NS \\
\hline
\end{tabular}

Data given as mean \pm SD or $\mathrm{n}(\%)$.

EPS, electrophysiological study; ERP, effective refractory period; iso, isoelectric; neg, negative; RFCA, radiofrequency catheter ablation; RVA, right ventricular apex. Other abbreviations as in Tables 1,2. 
A

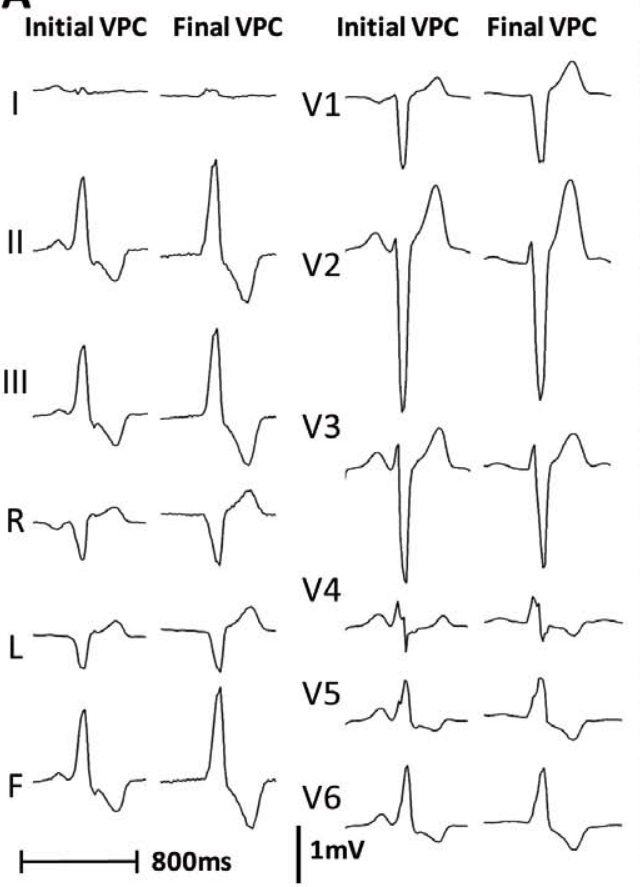

B
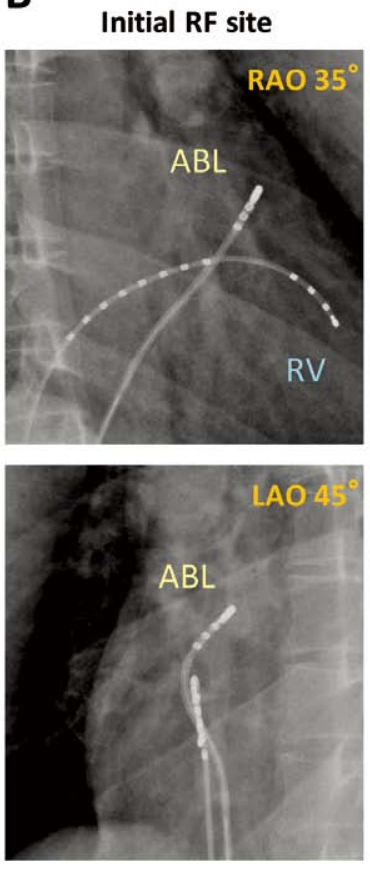

Final RF site
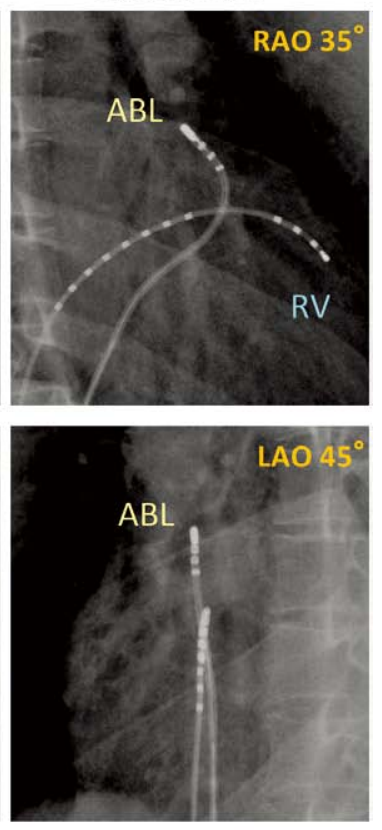

Figure 3. Representative case of QRS morphological change after radiofrequency (RF) energy application. Patient 6. (A) 12-lead electrocardiogram (ECG) before and after the initial RF energy application in the right ventricular outflow tract (RVOT). (B) Radiograms (right anterior oblique projection [RAO] $35^{\circ}$ and left anterior oblique projection [LAO] $45^{\circ}$ ) at (Left) the initial ablation site and (Right) final ablation site in the RVOT. In this case, the earliest ventricular activation site was initially located on the anterior attachment side of the septum. A ventricular premature contraction (VPC) with a positive QRS complex, however, appeared after the RF application at that site. Finally, an RF application at the posterior attachment side of the free wall in the RVOT successfully eliminated the residual VPC. ABL, ablation catheter; RV, right ventricle.

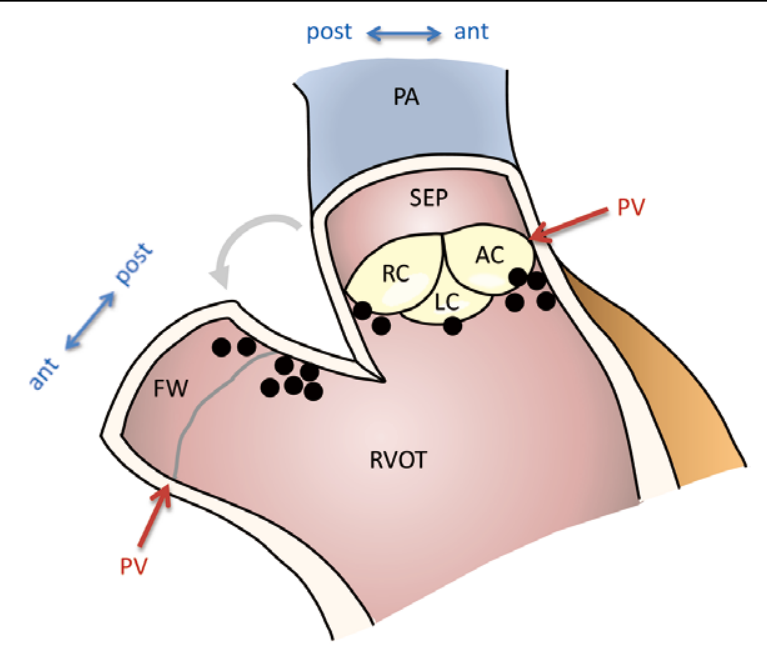

Figure 4. Distribution of the final successful ablation sites in 14 patients with ventricular fibrillation/polymorphic ventricular tachycardia (VF/PVT): coronal section image of the ventriculopulmonary junction. In 9 of 14 patients, the successful ablation sites were located on the posterior attachment of the right ventricular outflow tract (RVOT). AC, anterior cusp of pulmonary valve; ant, anterior attachment; FW, free wall; LC, left cusp; PA, pulmonary artery; post, posterior attachment; PV, level of pulmonary valve; RC, right cusp; SEP, septum. midway between the anterior and posterior attachment of the septum in 1, and at the anterior attachment of the septum in 4. The final successful ablation site for the VPCs was above the pulmonary valve in 2 patients $(14 \%)$ in the VF/PVT group and in 7 patients (9\%) in the MVT group. In all 9 patients with VPC originating above the pulmonary valve, a sharp potential preceding the QRS onset and a subsequent dull potential were recorded during the VPCs from the ablation catheter (Figure 5). The sharp potential was also eliminated after successful RF energy application. After a mean follow-up period of $71 \pm 34$ months, successful RFCA was achieved in 13 patients $(93 \%)$ in the VF/PVT group and in $65(84 \%)$ in the MVT group. Partial success (elimination of the VT with residual isolated VPCs from the RVOT) was obtained in 1 (7\%) and $10(13 \%)$ patients in the VF/PVT and MVT groups, respectively.

\section{Discussion}

\section{Major Findings}

The major findings are as follows: (1) a positive QRS complex of the initial VPC in lead I was observed in the majority of cases of VF/PVT; (2) syncopal episodes were more often observed in the VF/PVT group than in the MVT group; (3) no difference was observed in the coupling interval of the initial VPC between the 2 groups; and (4) the effect of RFCA for several years was favorable in the 2 groups.

These results indicate that idiopathic VF/PVT from the 


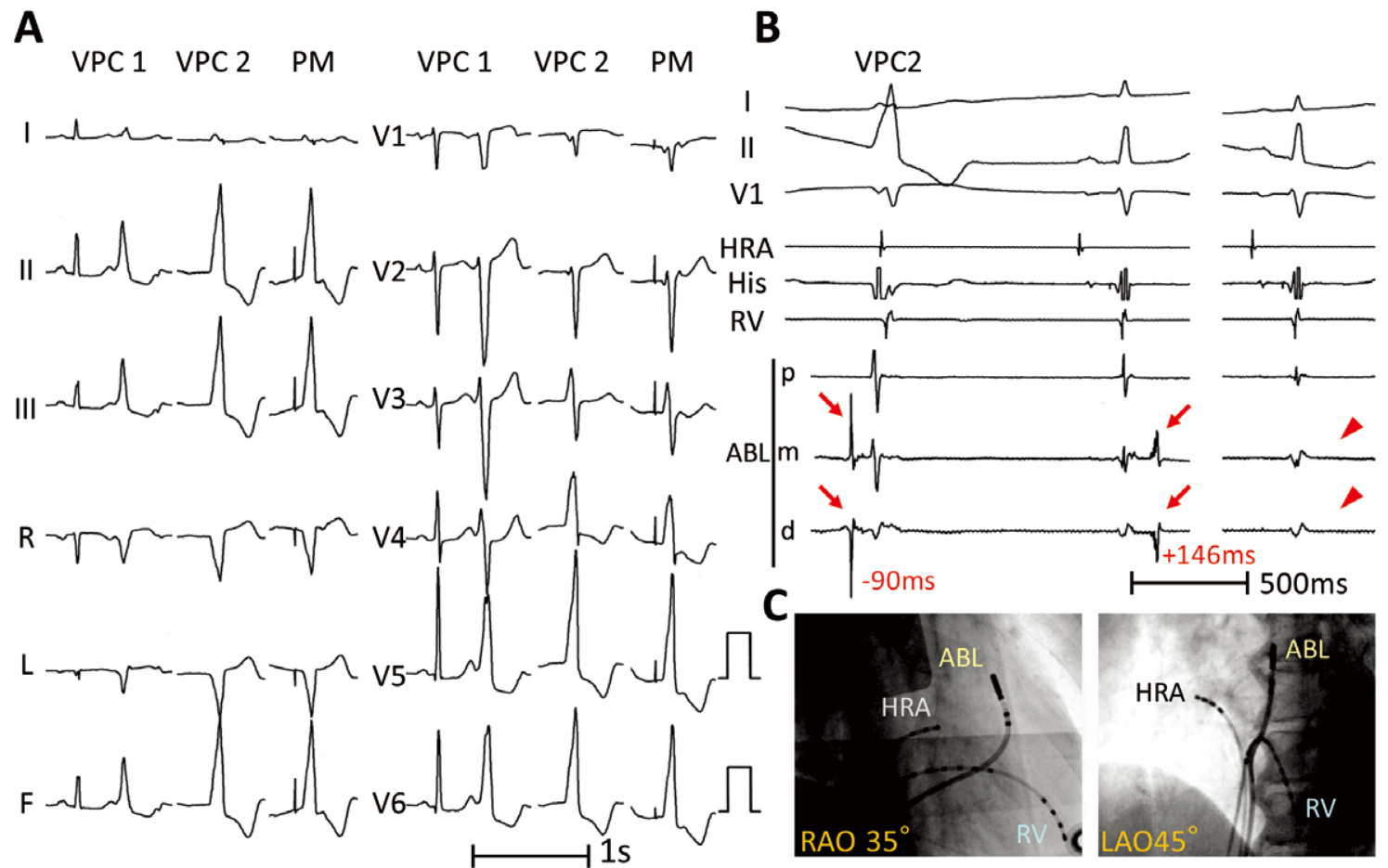

Figure 5. Representative case of ventricular fibrillation triggered by a ventricular premature contraction (VPC) arising from the pulmonary artery (PA). Patient 12. (A) QRS morphology of the triggering VPC at baseline (VPC1) and that after several radiofrequency (RF) applications to the right ventricular outflow tract (RVOT; VPC2). (B) Intracardiac electrograms (Left) before and (Right) after a successful RF application in the PA. (C) Radiograms (right anterior oblique projection [RAO] $35^{\circ}$ and left anterior oblique projection [LAO] $45^{\circ}$ ) at the successful ablation site in the PA. In this patient, VPC1 was observed at baseline. The earliest ventricular activation site of VPC1 was the posterior attachment side of the free wall below the pulmonary valve (PV). After several RF energy applications below the PV, a VPC with a slightly different QRS morphology (VPC2) occurred. A sharp potential (arrows in B) following the ventricular activation was recorded during the PA mapping. The QRS morphology during pace mapping (PM) in the PA was identical to that of VPC2. A sharp potential preceded the QRS onset by 90 ms during VPC2. During sinus rhythm, a sharp potential was recorded after the QRS (arrows in B). After the elimination of VPC2 by an RF energy application at the same site, the sharp potential after the QRS disappeared (arrowheads in B). ABL, ablation catheter; d, distal; HRA, high right atrium; $m$, middle; $p$, proximal; $R V$, right ventricle.

RVOT, although rare, should be considered when a patient has a syncopal episode and RVOT-VPC with a positive deflection of the QRS complex in lead I. Six (86\%) of 7 patients with syncope plus positive QRS in lead I had a malignant VA. The RF applications targeting the trigger VPC frequently caused a change in the QRS morphology, but the VF/PVT had been eliminated by additional ablation applications for residual VPCs.

\section{ECG Characteristics of the First VPC Initiating the PVT}

Although there are several case reports of RVOT-PVT, ${ }^{5-9}$ no report referred to the QRS morphology of the trigger VPC. In these reports, all of the first VPCs initiating the PVT had a positive deflection in lead I. In the present study, 10 of 14 patients with VF/PVT had a positive QRS complex in lead I before RFCA. The RVOT-VPCs with a positive QRS wave in lead I usually arise from the posterior attachment side of the septum or free wall. ${ }^{11}$ As shown in Figure 4, in 9 of 14 patients, the final successful ablation sites in the present study were also on the posterior attachment of the RVOT.

As shown in Figure 1, the coupling interval between the single VPC and the first VPC initiating PVT in patient 2 was not identical, but the coupling intervals of each VPC in the same individual were almost constant with or without subsequent VF/PVT. In addition, no significant difference was observed in the QRS morphology between the isolated VPC and trigger VPC of VF/PVT, and the coupling interval of trigger VPCs was similar to that of the RVOT-VPC in other common benign cases. We could not understand how the slight difference in the coupling interval as shown in Figure 1 is related to polymorphic QRS complex formation.

In this study, a morphological change in the targeted VPC was frequently observed during the RFCA sessions in the VF/ PVT group. Noda et al also reported that the alterations of QRS morphology were detected in the initial VPC of VF/PVT. ${ }^{3}$ As previously reported, the QRS morphological change during RFCA was considered to be a result of the shift of the activation pathways between the tachycardia focus and the exit point of the targeted VPC. ${ }^{12}$ Tada et al noted that morphological changes during RFCA were often observed in patients with VA arising from the pulmonary artery, and asserted that this phenomenon may be associated with the structure and distribution of the pulmonary myocardial extensions. ${ }^{13}$ A previous study using autopsy hearts found that only $17 \%$ of subjects with no association with arrhythmic events had a ventricular 
myocardial extension into the pulmonary artery beyond the ventriculo-arterial junction. ${ }^{14}$ In addition, the pulmonary myocardial extensions are widely distributed in the basal region of the pulmonary valve and taper at the distal pulmonary artery. $\mathrm{RF}$ applications at the basal region of the pulmonary valve result in changes in the tachycardia exit, and as a result, the morphology of the VPC may be changed during RF ablation. In the present study, 2 of 4 patients with VF had a tachycardia focus above the pulmonary valve. The pulmonary artery, especially at the posterior attachment site, might be a commonly affected site of VF.

\section{Possible Mechanism of VF/PVT}

The mechanism of benign idiopathic MVT from the RVOT is considered to be triggered activity; that of RVOT-VF/PVT is still unclear because it is difficult to induce VF/PVT by any ventricular burst pacing or ventricular programmed stimulation with or without catecholamines in the EP laboratory. Furthermore, even if the VF/PVT is inducible by ventricular stimulation, EPS during tachycardia is difficult due to its hemodynamic instability.

At baseline, no patients had any negative $\mathrm{T}$ waves in the precordial leads or late potentials in the SAECG. During mapping of the RVOT in sinus rhythm, neither any low-voltage area nor delayed potentials were recognized in any of the patients. No patients had any fibro-fatty changes in the RV on cardiac CT or MRI. The diagnosis of ARVC, however, can be difficult in the absence of any overt clinical abnormalities. Therefore, patients with early-phase ARVC might have been included.

In cases of VF/PVT reported previously, the QRS complex demonstrated a polymorphism during rapid ventricular pacing from the RV. In addition, burst pacing from the earliest activation site could reproduce several initial QRS complexes identical to the documented PVT. ${ }^{3,6}$ This reproducibility suggested that the PVT from the RVOT occurred from a single focus by triggered activity or micro-reentry with multiple myocardial exits to the RV and the development of polymorphic QRS waves.

All 14 patients with VF/PVT were previously diagnosed with isolated VPCs. The average period from VPC detection to clinical onset of VA was $120 \pm 118$ months. This indicates that VF/PVT developed at some point after an asymptomatic period of several years.

\section{Study Limitations}

The number of patients in the RVOT-VF/PVT group was small. In the 14 patients with VF/PVT, the QT prolongation and/or $\mathrm{T}$ wave alteration by isoproterenol was not observed during EPS and RFCA. It might be possible, however, that the latent form of long QT syndrome was included among the 14 patients.

Twelve $(86 \%)$ of 14 patients with VF/PVT were female in this study. A previous report indicated that RVOT-VA in women was 1.5 -fold more frequent than in men. ${ }^{15}$ Therefore, it is not known whether the malignant form of VPCs is frequent in female patients or not.

A positive QRS complex in lead I itself was common in the patients with isolated VPCs or MVT arising from the RVOT. A total of 27 of 77 patients in the MVT group also had a positive QRS in lead I in the present study. Many other individuals who did not undergo RFCA consisted of asymptomatic patients with isolated RVOT-VPCs. It is important to distinguish malignant VPCs from benign VPCs in many asymptomatic patients with isolated RVOT-VPCs, but it remains unknown how many patients with isolated VPCs with a possible malignant form will develop VF/PVT in the future.

\section{Conclusion}

Malignant VAs from the RVOT are rare. We should pay attention, however, to any syncopal episodes and VPCs with a positive deflection of the QRS complex in lead I.

\section{Acknowledgments}

Conflict of Interest: None.

\section{Disclosures}

No author has a real or perceived conflict of interest.

\section{References}

1. Haïssaguerre M, Shoda M, Jaïs P, Nogami A, Shah DC, Kautzner J, et al. Mapping and ablation of idiopathic ventricular fibrillation. Circulation 2002; 106: 962-967.

2. Viskin S, Rosso R, Rogowski O, Belhassen B. The "short-coupled" variant of right ventricular outflow ventricular tachycardia: A not-sobenign form of benign ventricular tachycardia? J Cardiovasc Electrophysiol 2005; 16: $912-916$.

3. Noda T, Shimizu W, Taguchi A, Aiba T, Satomi K, Suyama K, et al. Malignant entity of idiopathic ventricular fibrillation and polymorphic ventricular tachycardia initiated by premature extrasystoles originating from the right ventricular outflow tract. J Am Coll Cardiol 2005; 46: $1288-1294$.

4. Shimizu W. Arrhythmias originating from the right ventricular outflow tract: How to distinguish "malignant" from "benign"? Heart Rhythm 2009; 6: 1507-1511.

5. Uemura T, Yamabe H, Tanaka Y, Morihisa K, Kawano H, Kaikita K, et al. Catheter ablation of a polymorphic ventricular tachycardia inducing monofocal premature ventricular complex. Intern Med 2008; 47: $1799-1802$.

6. Kusano KF, Yamamoto M, Emori T, Morita H, Ohe T. Successful catheter ablation in a patient with polymorphic ventricular tachycardia. J Cardiovasc Electrophysiol 2000; 11: 682-685.

7. Takatsuki S, Mitamura H, Ogawa S. Catheter ablation of a monofocal premature ventricular complex triggering idiopathic ventricular fibrillation. Heart 2001; 86: e3.

8. Ashida K, Kaji Y, Sasaki Y. Abolition of torsade de pointes after radiofrequency catheter ablation at right ventricular outflow tract. Int J Cardiol 1997; 59: 171-175.

9. Kataoka M, Takatsuki S, Tanimoto K, Akaishi M, Ogawa S, Mitamura $\mathrm{H}$. A case of vagally mediated idiopathic ventricular fibrillation. Nat Clin Pract Cardiovasc Med 2008; 5: 111-115.

10. Tada H. Catheter ablation of tachyarrhythmias from the aortic sinuses of Valsalva: When and how? Circ J 2012; 76: 791-800.

11. Yoshida Y, Hirai M, Murakami Y, Kondo T, Inden Y, Akahoshi M, et al. Localization of precise origin of idiopathic ventricular tachycardia from the right ventricular outflow tract by a 12-lead ECG: A study of pace mapping using a multielectrode "basket" catheter. Pacing Clin Electrophysiol 1999; 22: 1760-1768.

12. Tada H, Hiratsuji T, Naito S, Kurosaki K, Ueda M, Ito S, et al. Prevalence and characteristics of idiopathic outflow tract tachycardia with QRS alteration following catheter ablation requiring additional radiofrequency ablation at a different point in the outflow tract. Pacing Clin Electrophysiol 2004; 9: 1240-1249.

13. Tada H, Tadokoro K, Miyaji K, Ito S, Kurosaki K, Kaseno K, et al. Idiopathic ventricular arrhythmias arising from the pulmonary artery: Prevalence, characteristics, and topography of the arrhythmia origin. Heart Rhythm 2008; 5: 419-426.

14. Hasdemir C, Aktas S, Govsa F, Aktas EO, Kocak A, Bozkaya YT, et al. Demonstration of ventricular myocardial extensions into the pulmonary artery and aorta beyond the ventriculo-arterial junction. Pacing Clin Electrophysiol 2007; 30: 534-539.

15. Tanaka Y, Tada H, Ito S, Naito S, Higuchi K, Kumagai K et al. Gender and age differences in candidates for radiofrequency catheter ablation of idiopathic ventricular arrhythmias. Circ J 2011; 75: 1585 1591. 